\title{
EDITORIAL
}

\section{Zu wenig Wasser oder zu wenig Zuversicht?}

\author{
Randolf Rausch · Johannes Barth
}

Betrachtet man heute die Medien, stößt man täglich auf neue Schreckensmeldungen zum Thema Wasser. Es gibt Warnungen zu einer lebensbedrohenden weltweiten Wasserkrise. Berichte von Dürren, Kriegen um Wasser, vergiftetem Wasser und dem Ende unserer Wasserressourcen schüren Ängste bald verdursten zu müssen oder kein Trinkwasser mehr zu haben.

Diese Meldungen entsprechen der Denkweise vieler Medien ,What bleeds leads“. Sie mögen wohl die Auflage der Zeitschriften bzw. die Einschaltquoten der TV-Sender erhöhen, tragen aber nichts zu einer seriösen Information bei, noch helfen sie bei der Lösung der Probleme.

Als Experten wissen wir, dass die Situation tatsächlich ernst ist und nicht auf die leichte Schulter genommen werden kann. Wir wissen aber auch, dass wir nicht resignieren müssen. In vielen Fällen besitzen wir schon heute Antwor- ten und passende Lösungen. Wir dürfen also die Information und Diskussion zum Thema Wasser nicht allein den Medien überlassen. Es ist an uns, die Sachverhalte verständlich, klar und wissenschaftlich korrekt darzustellen und Lösungsansätze zu entwickeln. Das Interview im Nachrichtenteil mit dem Titel „Wir müssen nicht verdursten - Wege aus der Wasserkrise" stellt einen Überblick über die aktuellen Herausforderungen auf dem Wassersektor dar, wobei das Grundwasser eine bedeutende Rolle spielt. Trotz aller Probleme lässt sich eine insgesamt positive Bilanz für die globale Wassersituation und zukünftige Prognosen ziehen. Das Thema Grundwasser ist dabei von allergrößter Bedeutung. Für uns Hydrogeologen ist es eine interessante und spannende Aufgabe sowie eine Herausforderung für die $\mathrm{Zu}$ kunft. Noch nie war unsere Expertise so nötig wie heute. Wir wünschen allen viel Vergnügen beim Lesen.

R. Rausch $(\bowtie)$

Gesellschaft für Internationale Zusammenarbeit (GIZ),

Riad, Saudi Arabien

E-Mail: Randolf.Rausch@gizdco.com

J. Barth

Lehrstuhl für Angewandte Geologie,

Friedrich-Alexander-Universität Erlangen-Nürnberg,

GeoZentrum Nordbayern,

Schlossgarten 5, 91054 Erlangen, Deutschland 